\title{
A hybrid kinetic WENO scheme for inviscid and viscous flows
}

\author{
Hongwei Liu*,† \\ State Key Laboratory of High Temperature Gas Dynamics, Institute of Mechanics, Chinese Academy of Sciences, \\ Beijing 100190, China
}

\begin{abstract}
SUMMARY
In this paper, we propose a high-order finite volume hybrid kinetic Weighted Essentially Non-Oscillatory (WENO) scheme for inviscid and viscous flows. Based on the WENO reconstruction technique, a hybrid kinetic numerical flux is introduced for the present method, which includes the mechanisms of both the free transfer and the collision of gas molecules. The collisionless free transfer part of the hybrid numerical flux is constructed from the conventional kinetic flux vector splitting treatment, and the collision contribution is considered by constructing an equilibrium gas state and calculating the corresponding numerical flux at the cell interface. The total variation diminishing Runge-Kutta methods are used for the temporal integration. The high-order accuracy and good shock-capturing capability of the proposed hybrid kinetic WENO scheme are validated by many numerical examples in one-dimensional and two-dimensional cases. Copyright (C) 2015 John Wiley \& Sons, Ltd.
\end{abstract}

Received 27 August 2014; Revised 20 April 2015; Accepted 9 May 2015

KEY WORDS: WENO schemes; kinetic numerical fluxes; numerical dissipations

\section{INTRODUCTION}

Essentially Non-Oscillatory (ENO) schemes were started with the classic paper of Harten et al. [1] and further efficiently implemented in [2, 3] for hyperbolic conservation laws. Later, Weighted ENO (WENO) schemes were developed $[4,5]$, using a convex combination of all candidate stencils instead of just one as in the original ENO idea. The WENO reconstruction is very effective in both controlling numerical oscillations and restoring smooth distributions, which has been widely used in many practical applications.

In recent years, the development of gas-kinetic schemes for compressible flow simulations has attracted much attention and become mature, such as the kinetic flux vector splitting (KFVS) methods [6-8], the various algorithms based on the Bhatnagar-Gross-Krook (BGK) model [9-13], and many others. The gas-kinetic schemes use various kinetic equations to model the dynamic processes around a cell interface and can provide robust and accurate numerical solutions for various compressible flows.

The combination of the WENO reconstruction and gas-kinetic flux formulation has been recently studied by some researchers [14-16]. In this paper, a new hybrid kinetic WENO scheme is proposed based on the hybridization of two types of kinetic fluxes, that is, the free transfer KFVS flux and the collision-related flux, both evaluated from the WENO reconstruction technique. The total variation diminishing (TVD) Runge-Kutta methods [2] are employed for the temporal integration in the present scheme. Both one-dimensional (1D) and two-dimensional (2D) test problems will be shown to evaluate the performance of the present hybrid kinetic WENO scheme.

\footnotetext{
*Correspondence to: Hongwei Liu, State Key Laboratory of High Temperature Gas Dynamics, Institute of Mechanics, Chinese Academy of Sciences, Beijing 100190, China.

†E-mail: hliu@imech.ac.cn 
The paper is organized as follows. In Section 2, we present the gas kinetic theory used in this paper. In Section 3, the newly developed hybrid kinetic WENO scheme will be described in detail. Numerical experiments are reported in Section 4 and conclusions are made in Section 5.

\section{EULER EQUATIONS AND GAS KINETIC THEORY}

The Euler equations for inviscid flows can be written as

$$
\frac{\partial \mathbf{U}}{\partial t}+\sum_{m=1}^{d} \frac{\partial \mathbf{F}_{m}(\mathbf{U})}{\partial x_{m}}=0
$$

where $d$ is the space dimension, $\mathbf{x}=\left(x_{1}, \cdots, x_{d}\right)$, the conservative vector $\mathbf{U}$ and the flux vector $\mathbf{F}_{m}(\mathbf{U})$ are given by

$$
\begin{aligned}
\mathbf{U} & =\left(\rho, \rho u_{1}, \cdots, \rho u_{d}, E\right)^{T}, \\
\mathbf{F}_{m}(\mathbf{U}) & =\left(\rho u_{m}, \rho u_{m} u_{1}+\delta_{m, 1} p, \cdots, \rho u_{m} u_{d}+\delta_{m, d} p, u_{m}(E+p)\right)^{T},
\end{aligned}
$$

where $\rho$ is the fluid density, $\mathbf{u}=\left(u_{1}, \cdots, u_{d}\right)^{T}$ is the velocity vector, $p$ is the pressure, $E=$ $\rho\left(e+\frac{1}{2} \mathbf{u}^{2}\right)$ is the total energy, and $\delta_{m, n}$ is the Kronecker delta. For an ideal gas, the thermal energy $e$ is related to the pressure $p$ through the relation

$$
p=(\gamma-1) \rho e,
$$

where $\gamma$ is the ratio of the specific heats.

In the gas kinetic theory, the basic evolution equation for the distribution function $f$ is the wellknown Boltzmann equation, which can be written as

$$
\frac{\partial f}{\partial t}+\sum_{m=1}^{d} v_{m} \frac{\partial f}{\partial x_{m}}=J(f, f),
$$

where $\mathbf{v}=\left(v_{1}, \cdots, v_{d}\right)^{T}$ denotes the particle velocity, the right-hand side (RHS) of Eq. (2.4) represents the particle collision term, which is in a complex integral form. The Maxwellian equilibrium distribution function $g$ reads

$$
g(\mathbf{x}, t, \mathbf{v}, \xi)=\rho\left(\frac{\lambda}{\pi}\right)^{\frac{K+d}{2}} e^{-\lambda\left((\mathbf{v}-\mathbf{u})^{2}+\xi^{2}\right)},
$$

where $(\mathbf{v}-\mathbf{u})^{2}=\sum_{m=1}^{d}\left(v_{m}-u_{m}\right)^{2}, \xi^{2}=\sum_{n=1}^{K} \xi_{n}^{2}, K$ is the total number of degrees of freedom for the internal degree of freedom variable $\xi, \lambda=M / 2 k_{B} T$, here $M$ denotes the particle mass, $k_{B}$ is the Boltzmann constant, and $T$ is the temperature. The relation between macroscopic variables and distribution functions is

$$
\mathbf{U}=\int_{\mathcal{R}^{K+d}} \psi f(\mathbf{x}, t, \mathbf{v}, \xi) d \mathbf{v} d \xi=\int_{\mathcal{R}^{K+d}} \psi g(\mathbf{x}, t, \mathbf{v}, \xi) d \mathbf{v} d \xi
$$

where $\psi$ denotes the vector of moments defined as

$$
\psi=\left(1, v_{1}, \cdots, v_{d}, \frac{1}{2}\left(\mathbf{v}^{2}+\xi^{2}\right)\right)^{T},
$$

and $d \mathbf{v} d \xi=d v_{1} \cdots d v_{d} d \xi_{1} \cdots d \xi_{K}$ denotes the volume element in the phase space. 
The collisionless Boltzmann equation can be obtained by replacing the distribution function $f$ with the Maxwellian distribution function $g$ in Eq. (2.4),

$$
\frac{\partial g}{\partial t}+\sum_{m=1}^{d} v_{m} \frac{\partial g}{\partial x_{m}}=0
$$

The Euler equations (2.1) can be gained by taking moments of $\psi$ to the earlier equation

$$
\frac{\partial}{\partial t} \int_{\mathcal{R}^{K+d}} \psi g(\mathbf{x}, t, \mathbf{v}, \xi) d \mathbf{v} d \xi+\sum_{m=1}^{d} \frac{\partial}{\partial x_{m}} \int_{\mathcal{R}^{K+d}} v_{m} \psi g(\mathbf{x}, t, \mathbf{v}, \xi) d \mathbf{v} d \xi=0 .
$$

For many kinetic methods, such as the KFVS-type schemes, the collisionless Boltzmann equation (2.8) is used in the gas evolution stage for the numerical flux evaluation.

\section{HYBRID KINETIC WEIGHTED ESSENTIALLY NON-OSCILLATORY SCHEME}

A finite volume hybrid kinetic WENO scheme, which combines the WENO reconstruction technique and the kinetic formulation, will be presented in detail in this section.

\subsection{Weighted essentially non-oscillatory reconstruction technique}

The WENO reconstruction technique proposed in [5] is briefly described in this subsection. Assume that $f$ is the variable that needs to be reconstructed, $\bar{f}_{i}$ is the cell-averaged value in the $i$ th cell of a uniform grid, and $\hat{f}_{i+\frac{1}{2}}^{(l)}$ and $\hat{f}_{i+\frac{1}{2}}^{(r)}$ are two values obtained from the reconstruction at left and right limits of the interface $x_{i+\frac{1}{2}}$. The evaluation of $\hat{f}_{i+\frac{1}{2}}^{(l)}$ using WENO technique will be presented in the following and $\hat{f}_{i+\frac{1}{2}}^{(r)}$ can be obtained by a symmetric procedure with respect to $x_{i+\frac{1}{2}}$. As shown in [5], the 5th-order WENO reconstruction for $\hat{f}_{i+\frac{1}{2}}^{(l)}$ can be written as

$$
\hat{f}_{i+\frac{1}{2}}^{(l)}=w_{1} \hat{f}_{i+\frac{1}{2}}^{(1)}+w_{2} \hat{f}_{i+\frac{1}{2}}^{(2)}+w_{3} \hat{f}_{i+\frac{1}{2}}^{(3)}
$$

where $\hat{f}_{i+\frac{1}{2}}^{(k)}$ are three third-order approximated values on three different stencils given by

$$
\begin{aligned}
& \hat{f}_{i+\frac{1}{2}}^{(1)}=\frac{1}{3} \bar{f}_{i-2}-\frac{7}{6} \bar{f}_{i-1}+\frac{11}{6} \bar{f}_{i}, \\
& \hat{f}_{i+\frac{1}{2}}^{(2)}=-\frac{1}{6} \bar{f}_{i-1}+\frac{5}{6} \bar{f}_{i}+\frac{1}{3} \bar{f}_{i+1}, \\
& \hat{f}_{i+\frac{1}{2}}^{(3)}=\frac{1}{3} \bar{f}_{i}+\frac{5}{6} \bar{f}_{i+1}-\frac{1}{6} \bar{f}_{i+2},
\end{aligned}
$$

and the nonlinear weights $w_{k}$ are given by

$$
w_{k}=\frac{\tilde{w}_{k}}{\sum_{n=1}^{3} \tilde{w}_{n}}, \quad \tilde{w}_{n}=\frac{\gamma_{n}}{\left(\varepsilon+\beta_{n}\right)^{2}},
$$

with the linear weights $\gamma_{n}$ given by

$$
\gamma_{1}=\frac{1}{10}, \quad \gamma_{2}=\frac{3}{5}, \quad \gamma_{3}=\frac{3}{10}
$$


and the smoothness indicators $\beta_{n}$ given by

$$
\begin{aligned}
& \beta_{1}=\frac{13}{12}\left(\bar{f}_{i-2}-2 \bar{f}_{i-1}+\bar{f}_{i}\right)^{2}+\frac{1}{4}\left(\bar{f}_{i-2}-4 \bar{f}_{i-1}+3 \bar{f}_{i}\right)^{2}, \\
& \beta_{2}=\frac{13}{12}\left(\bar{f}_{i-1}-2 \bar{f}_{i}+\bar{f}_{i+1}\right)^{2}+\frac{1}{4}\left(\bar{f}_{i-1}-\bar{f}_{i+1}\right)^{2}, \\
& \beta_{3}=\frac{13}{12}\left(\bar{f}_{i}-2 \bar{f}_{i+1}+\bar{f}_{i+2}\right)^{2}+\frac{1}{4}\left(3 \bar{f}_{i}-4 \bar{f}_{i+1}+\bar{f}_{i+2}\right)^{2} .
\end{aligned}
$$

In Eq. (3.3), the parameter $\varepsilon$ is set to be positive to avoid the denominator to become zero and is taken as $\varepsilon=10^{-6}$ in this paper.

Recently, some improved smoothness indicators have been proposed and investigated [17-19]. In this paper, we will mainly use the smoothness indicator by Jiang and Shu [5], that is, Eqs. (3.3)(3.5), for the present hybrid kinetic WENO scheme. Our numerical experiments indicate that other smoothness indicators [17-19] can also work well for the proposed scheme; an illustration will be shown in Example 4.3.

\subsection{Hybrid kinetic weighted essentially non-oscillatory scheme for inviscid flows}

This section will introduce a hybrid kinetic WENO scheme for solving the Euler equations. For the sake of simplicity, only the one-dimensional case is presented and it can be easily extended to multidimensional cases in a dimension by dimension manner [5]. The 1D Euler equations can be written as

$$
\frac{\partial \mathbf{U}}{\partial t}+\frac{\partial \mathbf{F}(\mathbf{U})}{\partial x}=0
$$

where $\mathbf{U}=(\rho, \rho u, E)^{T}$ and $\mathbf{F}(\mathbf{U})$ is given by

$$
\mathbf{F}(\mathbf{U})=\left(\rho u, \rho u^{2}+p, u(E+p)\right)^{T} .
$$

For a uniform grid with the cell center $x_{i}$, the cell interface $x_{i+\frac{1}{2}}$, and the cell size $\Delta x$, a finite volume method can be written as

$$
\frac{d \overline{\mathbf{U}}_{i}}{d t}=-\frac{1}{\Delta x}\left(\hat{\mathbf{F}}_{i+\frac{1}{2}}-\hat{\mathbf{F}}_{i-\frac{1}{2}}\right),
$$

where $\overline{\mathbf{U}}_{i}$ is the cell-averaged value and $\hat{\mathbf{F}}_{i+\frac{1}{2}}$ is the numerical flux at cell interface $x_{i+\frac{1}{2}}$.

In this paper, the third-order TVD Runge-Kutta method proposed in [2] will be used to integrate Eq. (3.8) in time. Therefore, we only need to specify the construction of the numerical flux $\hat{\mathbf{F}}_{i+\frac{1}{2}}$ for a finite volume scheme.

In the following, first we will briefly describe the conventional WENO scheme [5], which uses only the flux splitting approach to evaluate the numerical flux, and then the hybrid kinetic WENO scheme will be introduced in detail.

3.2.1. Conventional weighted essentially non-oscillatory scheme. As shown in [5], the numerical flux $\hat{\mathbf{F}}_{i+\frac{1}{2}}$ in Eq. (3.8) can be divided into two parts:

$$
\hat{\mathbf{F}}_{i+\frac{1}{2}}=\hat{\mathbf{F}}_{i+\frac{1}{2}}^{+}+\hat{\mathbf{F}}_{i+\frac{1}{2}}^{-}
$$


where $\hat{\mathbf{F}}_{i+\frac{1}{2}}^{+}$is the flux along $x$-positive direction and $\hat{\mathbf{F}}_{i+\frac{1}{2}}^{-}$is the flux along $x$-negative direction. Let $\overline{\mathbf{F}}_{i}\left(\overline{\mathbf{U}}_{i}\right)$ be the numerical flux based on the cell-averaged value $\overline{\mathbf{U}}_{i}$. In order to evaluate $\hat{\mathbf{F}}_{i+\frac{1}{2}}^{ \pm}$in Eq. (3.9), first, we split the numerical flux $\overline{\mathbf{F}}_{i}$ into two parts:

$$
\overline{\mathbf{F}}_{i}=\overline{\mathbf{F}}_{i}^{+}+\overline{\mathbf{F}}_{i}^{-},
$$

which can be achieved by many flux splitting approaches, such as the Lax-Friedrichs [5] or Steger-Warming [20] flux splitting method. Then, the numerical flux $\hat{\mathbf{F}}_{i+\frac{1}{2}}^{+}$in Eq. (3.9) can be obtained from $\overline{\mathbf{F}}_{i}^{+}$by the fifth-order WENO reconstruction technique of Eq. (3.1), and $\hat{\mathbf{F}}_{i+\frac{1}{2}}^{-}$can be calculated from $\overline{\mathbf{F}}_{i}^{-}$by a symmetric procedure with respect to $x_{i+\frac{1}{2}}$. Finally, the numerical flux $\hat{\mathbf{F}}_{i+\frac{1}{2}}$ for a conventional WENO scheme [5] can be gained by substituting $\hat{\mathbf{F}}_{i+\frac{1}{2}}^{ \pm}$into Eq. (3.9). The underlying physical principle for such a conventional WENO algorithm is the collisionless free transfer of gas molecules.

For a kinetic scheme, $\overline{\mathbf{F}}_{i}^{ \pm}$in Eq. (3.10) can be obtained from the KFVS technique,

$$
\begin{aligned}
& \overline{\mathbf{F}}_{i}^{+}=\int_{\mathcal{R}^{K}} \int_{v>0} v \psi g\left(\overline{\mathbf{U}}_{i}, v, \xi\right) d v d \xi, \\
& \overline{\mathbf{F}}_{i}^{-}=\int_{\mathcal{R}^{K}} \int_{v<0} v \psi g\left(\overline{\mathbf{U}}_{i}, v, \xi\right) d v d \xi,
\end{aligned}
$$

where $\psi$ is defined as

$$
\psi=\left(1, v, \frac{1}{2}\left(v^{2}+\xi^{2}\right)\right)^{T},
$$

and $g\left(\overline{\mathbf{U}}_{i}, v, \xi\right)$ is the Maxwellian equilibrium distribution function corresponding to the cellaveraged value $\overline{\mathbf{U}}_{i}$.

The numerical flux $\hat{\mathbf{F}}_{i+\frac{1}{2}}$ in Eq. (3.9) calculated by the KFVS approach is denoted by $\hat{\mathbf{F}}_{i+\frac{1}{2}}^{\mathrm{KFVS}}$, and a conventional fifth-order WENO algorithm [5] based on the KFVS technique will be called the W5-KFVS scheme hereafter in this paper.

3.2.2. Hybrid kinetic weighted essentially non-oscillatory scheme. In the following, we will present the construction of a hybrid kinetic numerical flux, which includes the effects of both the free transfer and the collision of gas molecules. The hybrid kinetic flux can be written as

$$
\hat{\mathbf{F}}_{i+\frac{1}{2}}=\alpha \hat{\mathbf{F}}_{i+\frac{1}{2}}^{\mathrm{KFVS}}+(1-\alpha) \hat{\mathbf{F}}_{i+\frac{1}{2}}^{\mathrm{C}},
$$

where $\hat{\mathbf{F}}_{i+\frac{1}{2}}^{\mathrm{KFVS}}$ is the collisionless KFVS-type numerical flux, $\hat{\mathbf{F}}_{i+\frac{1}{2}}^{\mathrm{C}}$ is the numerical flux due to molecule collision effects, and $\alpha$ is a parameter in the range $0 \leqslant \alpha \leqslant 1$ and will be hereafter called the jump indicator. It should be pointed out that this kind of hybrid numerical fluxes has been used in some kinetic schemes, see for example [21-23], and to name just a few.

Because the evaluation of the collisionless KFVS-type flux $\hat{\mathbf{F}}_{i+\frac{1}{2}}^{\mathrm{KFVS}}$ in Eq. (3.13) is the same as that described in the preceding subsection, that is, Eqs. (3.9)-(3.12), therefore, in order to use Eq. (3.13) to get the hybrid numerical flux, we only need to determine the collision-related kinetic flux $\hat{\mathbf{F}}_{i+\frac{1}{2}}^{\mathrm{C}}$ and the jump indicator $\alpha$.

The collision-related kinetic flux $\hat{\mathbf{F}}_{i+\frac{1}{2}}^{\mathrm{C}}$ can be constructed as follows. The basic idea of evaluating $\hat{\mathbf{F}}_{i+\frac{1}{2}}^{\mathrm{C}}$ is to calculate the flux by Eq. (3.7) based on the collision-related state $\hat{\mathbf{U}}_{i+\frac{1}{2}}^{\mathrm{C}}$ constructed at the cell interface $x_{i+\frac{1}{2}}$,

$$
\hat{\mathbf{F}}_{i+\frac{1}{2}}^{\mathrm{C}}=\mathbf{F}\left(\hat{\mathbf{U}}_{i+\frac{1}{2}}^{\mathrm{C}}\right)
$$


In order to get the collision-related state $\hat{\mathbf{U}}_{i+\frac{1}{2}}^{C}$, first we split the cell-averaged conservative variable $\overline{\mathbf{U}}_{i}$ into two parts:

$$
\overline{\mathbf{U}}_{i}=\overline{\mathbf{U}}_{i}^{+}+\overline{\mathbf{U}}_{i}^{-}
$$

with

$$
\begin{aligned}
& \overline{\mathbf{U}}_{i}^{+}=\int_{\mathcal{R}^{K}} \int_{v>0} \psi g\left(\overline{\mathbf{U}}_{i}, v, \xi\right) d v d \xi, \\
& \overline{\mathbf{U}}_{i}^{-}=\int_{\mathcal{R}^{K}} \int_{v<0} \psi g\left(\overline{\mathbf{U}}_{i}, v, \xi\right) d v d \xi,
\end{aligned}
$$

where $\psi$ is defined by Eq. (3.12) and $g\left(\overline{\mathbf{U}}_{i}, v, \xi\right)$ is the Maxwellian equilibrium distribution function corresponding to the cell-averaged value $\overline{\mathbf{U}}_{i}$.

Then, the collision-related state $\hat{\mathbf{U}}_{i+\frac{1}{2}}^{\mathrm{C}}$ at the cell interface $x_{i+\frac{1}{2}}$ can be obtained by

$$
\hat{\mathbf{U}}_{i+\frac{1}{2}}^{\mathrm{C}}=\hat{\mathbf{U}}_{i+\frac{1}{2}}^{+}+\hat{\mathbf{U}}_{i+\frac{1}{2}}^{-},
$$

where $\hat{\mathbf{U}}_{i+\frac{1}{2}}^{+}$is determined from $\overline{\mathbf{U}}_{i}^{+}$by the fifth-order WENO reconstruction technique, that is, Eq. (3.1), and $\hat{\mathbf{U}}_{i+\frac{1}{2}}^{-}$is calculated from $\overline{\mathbf{U}}_{i}^{-}$by a symmetric procedure with respect to $x_{i+\frac{1}{2}}$.

After $\hat{\mathbf{U}}_{i+\frac{1}{2}}^{\mathrm{C}}$ is determined by Eq. (3.17), the collision-related numerical flux $\hat{\mathbf{F}}_{i+\frac{1}{2}}^{\mathrm{C}}$ can be evaluated by Eq. (3.14).

Next, we consider the evaluation of the jump indicator $\alpha$. The principle to construct the jump indicator $\alpha$ is that the contribution of the flux $\hat{\mathbf{F}}_{i+\frac{1}{2}}^{\mathrm{KFVS}}$ should be dominant around strong shock waves and small in smooth regions. This is because the collisionless flux $\hat{\mathbf{F}}_{i+\frac{1}{2}}^{\mathrm{KFVS}}$ is more dissipative than the collision-related flux $\hat{\mathbf{F}}_{i+\frac{1}{2}}^{\mathrm{C}}$. Similar to the way in [21-23], in the present study, we use the local pressure jump around the cell interface to determine the jump indicator $\alpha$ in Eq. (3.13),

$$
\alpha=1-\exp \left(-C \frac{\left|\bar{p}_{i}-\bar{p}_{i+1}\right|}{\bar{p}_{i}+\bar{p}_{i+1}}\right)
$$

where $\bar{p}_{i}$ and $\bar{p}_{i+1}$ are pressures corresponding to cell-averaged values $\overline{\mathbf{U}}_{i}$ and $\overline{\mathbf{U}}_{i+1}$, respectively, and $C$ is an empirical positive constant. It can be seen that for the same local pressure jump, a larger value of $C$ results in a larger value of $\alpha$ and therefore more KFVS-type contribution in the hybrid kinetic flux, which makes the scheme more dissipative. Fortunately, our numerical experiments as well as those in [21-23] indicate that the numerical results are not sensitive to the chosen value of $C$; for the present hybrid kinetic scheme, we find that $C=10$ is a quite good choice based on plenty of numerical experiments; therefore, it is used for all the numerical tests in this paper, and the same value of $C$ has been adopted in [22].

From Eq. (3.13), we can see that both $\hat{\mathbf{F}}_{i+\frac{1}{2}}^{\mathrm{KFVS}}$ and $\hat{\mathbf{F}}_{i+\frac{1}{2}}^{\mathrm{C}}$ need to be calculated for each cell interface except for $\alpha=0$ or $\alpha=1$. In order to improve the efficiency of the proposed scheme, we introduce the following cut-off type of hybrid flux

$$
\hat{\mathbf{F}}_{i+\frac{1}{2}}=\left\{\begin{array}{lll}
\hat{\mathbf{F}}_{i+\frac{1}{2}}^{\mathrm{C}}, & \text { if } & 0 \leqslant \alpha \leqslant \delta, \\
\alpha \hat{\mathbf{F}}_{i+\frac{1}{2}}^{\mathrm{KFVS}}+(1-\alpha) \hat{\mathbf{F}}_{i+\frac{1}{2}}^{\mathrm{C}}, & \text { if } & \delta<\alpha<1-\delta, \\
\hat{\mathbf{F}}_{i+\frac{1}{2}}^{\mathrm{KFV}}, & \text { if } & 1-\delta \leqslant \alpha \leqslant 1,
\end{array}\right.
$$

where $\delta(0 \leqslant \delta<0.5)$ is a parameter to control the cut-off range of $\alpha$. Equation (3.19) is more efficient than Eq. (3.13) because the total number of cell interfaces for the former where both $\hat{\mathbf{F}}_{i+\frac{1}{2}}^{\mathrm{KFVS}}$ 
and $\hat{\mathbf{F}}_{i+\frac{1}{2}}^{\mathrm{C}}$ need to be evaluated is less than that for the latter. From Eq. (3.19), we can see that as the value of $\delta$ is increased, on the one hand, the efficiency of the scheme can be improved, but on the other hand the smoothness of the transition for the hybrid flux is reduced and therefore the numerical results usually become worse. So, we need to choose a suitable value of $\delta$ through numerical experiments; based on a large number of trials, we find that $\delta=0.02$ is a satisfactory choice after considering the overall performance, such as the accuracy and efficiency, of the proposed scheme, and therefore it is adopted in this paper. Moreover, it is observed that the numerical results are not sensitive to the value of $\delta$, for example, Eq. (3.19) with $\delta=0$ (i.e., Eq. (3.13)) and $\delta=0.02$ produces very similar numerical results in all the test problems; however, in terms of their efficiencies, the computational time for the former is roughly $50 \%$ more than that for the latter for most tests.

In this paper, Eq. (3.19) instead of Eq. (3.13) will be used for the newly proposed hybrid kinetic WENO scheme. Up to now, we have evaluated all the unknowns in Eq. (3.19), which can be used to get numerical fluxes for the finite volume scheme, that is, Eq. (3.8). Although the component by component version of the introduced hybrid kinetic WENO scheme is effective and works reasonably well for many problems, in this paper, we will use the more costly, but much more robust characteristic decomposition technique [5] in order to test some demanding problems.

The whole construction procedure of the numerical flux $\hat{\mathbf{F}}_{i+\frac{1}{2}}$ using Eq. (3.19) for the proposed hybrid kinetic WENO scheme can be summarized as the following steps:

1. Use the Roe average [24] of variables $\overline{\mathbf{U}}_{i}$ and $\overline{\mathbf{U}}_{i+1}$ to calculate the left and right eigenvector matrixes $\mathbf{L}_{i+\frac{1}{2}}$ and $\mathbf{R}_{i+\frac{1}{2}}$.

2. Compute the jump indicator $\alpha$ by Eq. (3.18), the following steps (3)-(5) are for the case $\delta<$ $\alpha<1-\delta$, similarly those for cases $0 \leqslant \alpha \leqslant \delta$ and $1-\delta \leqslant \alpha \leqslant 1$ can be easily obtained, which are omitted here.

3. Transform all the variables $\overline{\mathbf{F}}_{i+l}^{ \pm}, \overline{\mathbf{U}}_{i+l}^{ \pm}(l=-2, \ldots, 3)$ defined by Eqs. (3.11) and (3.16), respectively into the local characteristic fields

$$
\tilde{\mathbf{F}}_{i+l}^{ \pm}=\mathbf{L}_{i+\frac{1}{2}} \overline{\mathbf{F}}_{i+l}^{ \pm}, \quad \tilde{\mathbf{U}}_{i+l}^{ \pm}=\mathbf{L}_{i+\frac{1}{2}} \overline{\mathbf{U}}_{i+l}^{ \pm} .
$$

4. Perform the WENO reconstruction procedure [5] for each component of the characteristic variables $\tilde{\mathbf{F}}_{i+l}^{ \pm}$and $\tilde{\mathbf{U}}_{i+l}^{ \pm}$to obtain the corresponding component of $\hat{\tilde{\mathbf{F}}}_{i+\frac{1}{2}}^{ \pm}$and $\hat{\tilde{\mathbf{U}}}_{i+\frac{1}{2}}^{ \pm}$at the cell interface $x_{i+\frac{1}{2}}$.

5. Transform $\hat{\tilde{\mathbf{F}}}_{i+\frac{1}{2}}^{ \pm}$and $\hat{\tilde{\mathbf{U}}}_{i+\frac{1}{2}}^{ \pm}$back into the physical space

$$
\hat{\mathbf{F}}_{i+\frac{1}{2}}^{\mathrm{KFVS}}=\mathbf{R}_{i+\frac{1}{2}}\left(\hat{\tilde{\mathbf{F}}}_{i+\frac{1}{2}}^{+}+\hat{\tilde{\mathbf{F}}}_{i+\frac{1}{2}}^{-}\right), \quad \hat{\mathbf{U}}_{i+\frac{1}{2}}^{\mathrm{C}}=\mathbf{R}_{i+\frac{1}{2}}\left(\hat{\tilde{\mathbf{U}}}_{i+\frac{1}{2}}^{+}+\hat{\tilde{\mathbf{U}}}_{i+\frac{1}{2}}^{-}\right) .
$$

Calculate $\hat{\mathbf{F}}_{i+\frac{1}{2}}^{\mathrm{C}}$ based on $\hat{\mathbf{U}}_{i+\frac{1}{2}}^{\mathrm{C}}$ by Eq. (3.14).

6. Use Eq. (3.19) to obtain the hybrid numerical flux $\hat{\mathbf{F}}_{i+\frac{1}{2}}$.

It should be pointed out that if the collision-related state $\hat{\mathbf{U}}_{i+\frac{1}{2}}^{C}$ in Eq. (3.14) is constructed from a smooth reconstruction, such as the central interpolation from $\overline{\mathbf{U}}_{i}$, the resulting scheme does not work well. Similar observations have been reported in [22]. This means that the collisionless flux $\hat{\mathbf{F}}_{i+\frac{1}{2}}^{\mathrm{KFVS}}$ is very dissipative, and the collision-related flux $\hat{\mathbf{F}}_{i+\frac{1}{2}}^{\mathrm{C}}$ corresponding to $\hat{\mathbf{U}}_{i+\frac{1}{2}}^{\mathrm{C}}$ given by Eq. (3.17) is less dissipative than $\hat{\mathbf{F}}_{i+\frac{1}{2}}^{\mathrm{KFVS}}$, but more dissipative than that corresponding to $\hat{\mathbf{U}}_{i+\frac{1}{2}}^{\mathrm{C}}$ given by a smooth reconstruction. Therefore, the kinetic reconstruction of Eq. (3.17) is the key for the successful hybridization of different numerical fluxes.

The newly proposed fifth-order hybrid kinetic WENO algorithm will be called the W5-HK scheme hereafter in this paper. 


\subsection{Extension to viscous flows}

For viscous flows governed by the Navier-Stokes equations, the conventional central differencing is used for the viscous flux terms in the present method. For example, for the two-dimensional case with $\mathbf{x}=\left(x_{1}, x_{2}\right) \equiv(x, y)$, in calculating the $x$-direction viscous flux terms, we use the following sixth-order central differencing formulae to obtain the derivatives,

$$
\left(\frac{\partial q}{\partial x}\right)_{i+\frac{1}{2}, j}=\frac{2250\left(q_{i+1, j}-q_{i, j}\right)-125\left(q_{i+2, j}-q_{i-1, j}\right)+9\left(q_{i+3, j}-q_{i-2, j}\right)}{1920 \Delta x},
$$

and $\left(\frac{\partial q}{\partial x}\right)_{i-\frac{1}{2}, j}$ can be got through replacing $i$ by $i-1$ in Eq. (3.22),

$$
\left(\frac{\partial q}{\partial y}\right)_{i \pm \frac{1}{2}, j}=\frac{45\left(\hat{q}_{ \pm, j+1}-\hat{q}_{ \pm, j-1}\right)-9\left(\hat{q}_{ \pm, j+2}-\hat{q}_{ \pm, j-2}\right)+\left(\hat{q}_{ \pm, j+3}-\hat{q}_{ \pm, j-3}\right)}{60 \Delta y}
$$

where $\hat{q}_{+, j \pm l}$ and $\hat{q}_{-, j \pm l}(l=1,2,3)$ are defined by $\hat{q}_{+, j \pm l}=\left(q_{i, j \pm l}+q_{i+1, j \pm l}\right) / 2$ and $\hat{q}_{-, j \pm l}=$ $\left(q_{i-1, j \pm l}+q_{i, j \pm l}\right) / 2$, respectively. The variables at the cell interface needed for calculating viscous flux terms are gained by

$$
q_{i+\frac{1}{2}, j}=\frac{37\left(q_{i, j}+q_{i+1, j}\right)-8\left(q_{i-1, j}+q_{i+2, j}\right)+\left(q_{i-2, j}+q_{i+3, j}\right)}{60},
$$

and $q_{i-\frac{1}{2}, j}$ can be obtained through replacing $i$ by $i-1$ in Eq. (3.24).

\section{NUMERICAL EXPERIMENTS}

In this section, we will show some numerical examples in both one-dimensional and twodimensional cases. The uniform mesh is used for both $1 \mathrm{D}$ and $2 \mathrm{D}$ test problems. The computational time step $\Delta t$ is determined by [25]

$$
\Delta t=\left|\frac{\eta \Delta_{L}}{(|\mathbf{u}|+a)\left(1+R e^{*}\right)}\right|_{\min },
$$

where $\Delta_{L}$ is the representative length defined by $\Delta_{L}=\Delta x$ for $1 \mathrm{D}$ case and $\Delta_{L}=\min \{\Delta x, \Delta y\}$ for 2D case, $a$ is the speed of sound, $R e^{*}$ is the grid Reynolds number that is set to be zero for inviscid flows and $R e^{*}=|\mathbf{u}| \Delta_{L} / v$ for viscous flows with $v$ denoting the kinematic viscosity coefficient, and $\eta$ is the CFL number which is set to be $\eta=0.8$ unless specified in the present study. In Eq. (4.1), all the physical variables $\mathbf{u}, a, v$ use cell-averaged values and the minimum is taken over the whole computational domain.

\subsection{Inviscid flow problems}

\subsubsection{One-dimensional cases.}

Example 4.1 (1D accuracy test)

The first example is to test the accuracy of the method for 1D problems. We solve the 1D Euler equations with the initial data

$$
\rho(x, 0)=1+0.2 \sin (\pi x), \quad u(x, 0)=0.7, \quad p(x, 0)=1 .
$$


The computational domain is taken as [0,2], and the periodic boundary condition is used. The exact solution for this problem is given by

$$
\rho(x, t)=1+0.2 \sin (\pi(x-u t)), \quad u(x, t)=0.7, \quad p(x, t)=1 .
$$

We compute the solution up to $t=2$ with a suitably reduced time step to guarantee that spatial errors dominate.

The errors and convergence orders of density $\rho$ by the W5-KFVS and the W5-HK schemes are shown in Table I. This table shows that the fifth-order convergence rate can be obtained by both schemes. Moreover, the W5-HK has smaller absolute errors than the W5-KFVS given the same cell size; this means that the former is more accurate and less dissipative than the latter.

Example 4.2 (Blast wave problem)

The blast wave problem was originally proposed in [26], which is a challenging test case because of the complex flow structures. The initial flow field is given by

$$
(\rho, u, p)=\left\{\begin{array}{l}
(1,0,1000),-5 \leqslant x<-4 \\
(1,0,0.01),-4 \leqslant x<4 \\
(1,0,100), 4 \leqslant x \leqslant 5
\end{array}\right.
$$

The computational domain is $[-5,5]$ with a reflecting boundary condition on both ends.

Because the exact solution is unknown for this problem, the reference solution obtained by the W5-HK scheme with 10000 cells is used for comparison. The numerical results at $t=0.38$ are shown in Figure 1. From the figure, we can see that the W5-HK does perform better than the W5KFVS, especially for the complex flow regions.

\subsubsection{Two-dimensional cases.}

Example 4.3 (2D accuracy test)

This example is to test the accuracy of the hybrid kinetic WENO scheme for 2D problems. We solve the 2D Euler equations with the following initial data:

$$
\rho(x, y, 0)=1+0.2 \sin (\pi(x+y)), \quad u_{1}=0.7, \quad u_{2}=0.3, \quad p=1 .
$$

The computational domain is taken as $(x, y) \in[0,2] \times[0,2]$, and the periodic boundary condition is used along both $x$ and $y$ directions. The exact solution for this problem is

$$
\rho(x, y, t)=1+0.2 \sin (\pi(x+y-t)), \quad u_{1}=0.7, \quad u_{2}=0.3, \quad p=1 .
$$

Table I. One-dimentional accuracy test.

\begin{tabular}{llcccccc}
\hline \multirow{2}{*}{$N$} & Scheme & $L_{\text {error }}^{\infty}$ & Order & $L_{\text {error }}^{1}$ & Order & $L_{\text {error }}^{2}$ & Order \\
\hline \multirow{2}{*}{8} & W5-KFVS & $2.31 \mathrm{E}-2$ & - & $1.53 \mathrm{E}-2$ & - & $1.70 \mathrm{E}-2$ & - \\
& W5-HK & $2.08 \mathrm{E}-2$ & - & $1.08 \mathrm{E}-2$ & - & $1.33 \mathrm{E}-2$ & - \\
\multirow{2}{*}{16} & W5-KFVS & $1.66 \mathrm{E}-3$ & 3.80 & $9.57 \mathrm{E}-4$ & 4.00 & $1.06 \mathrm{E}-3$ & 4.00 \\
& W5-HK & $1.58 \mathrm{E}-3$ & 3.72 & $7.87 \mathrm{E}-4$ & 3.78 & $9.02 \mathrm{E}-4$ & 3.88 \\
\multirow{2}{*}{32} & W5-KFVS & $8.69 \mathrm{E}-5$ & 4.26 & $3.37 \mathrm{E}-5$ & 4.83 & $3.97 \mathrm{E}-5$ & 4.74 \\
& W5-HK & $6.10 \mathrm{E}-5$ & 4.69 & $2.71 \mathrm{E}-5$ & 4.86 & $3.17 \mathrm{E}-5$ & 4.83 \\
64 & W5-KFVS & $3.55 \mathrm{E}-6$ & 4.61 & $1.07 \mathrm{E}-6$ & 4.98 & $1.36 \mathrm{E}-6$ & 4.87 \\
& W5-HK & $1.94 \mathrm{E}-6$ & 4.97 & $8.26 \mathrm{E}-7$ & 5.04 & $9.66 \mathrm{E}-7$ & 5.04 \\
\multirow{2}{*}{128} & W5-KFVS & $1.16 \mathrm{E}-7$ & 4.94 & $3.34 \mathrm{E}-8$ & 5.00 & $4.23 \mathrm{E}-8$ & 5.01 \\
& W5-HK & $6.04 \mathrm{E}-8$ & 5.01 & $2.50 \mathrm{E}-8$ & 5.05 & $2.93 \mathrm{E}-8$ & 5.04 \\
\multirow{2}{*}{512} & W5-KFVS & $3.08 \mathrm{E}-9$ & 5.24 & $1.00 \mathrm{E}-9$ & 5.06 & $1.24 \mathrm{E}-9$ & 5.09 \\
& W5-HK & $1.76 \mathrm{E}-9$ & 5.10 & $7.78 \mathrm{E}-10$ & 5.01 & $8.96 \mathrm{E}-10$ & 5.03 \\
& W5-KFVS & $8.66 \mathrm{E}-11$ & 5.15 & $2.97 \mathrm{E}-11$ & 5.07 & $3.66 \mathrm{E}-11$ & 5.08 \\
& W5-HK & $5.06 \mathrm{E}-11$ & 5.12 & $2.47 \mathrm{E}-11$ & 4.98 & $2.79 \mathrm{E}-11$ & 5.01 \\
\hline
\end{tabular}



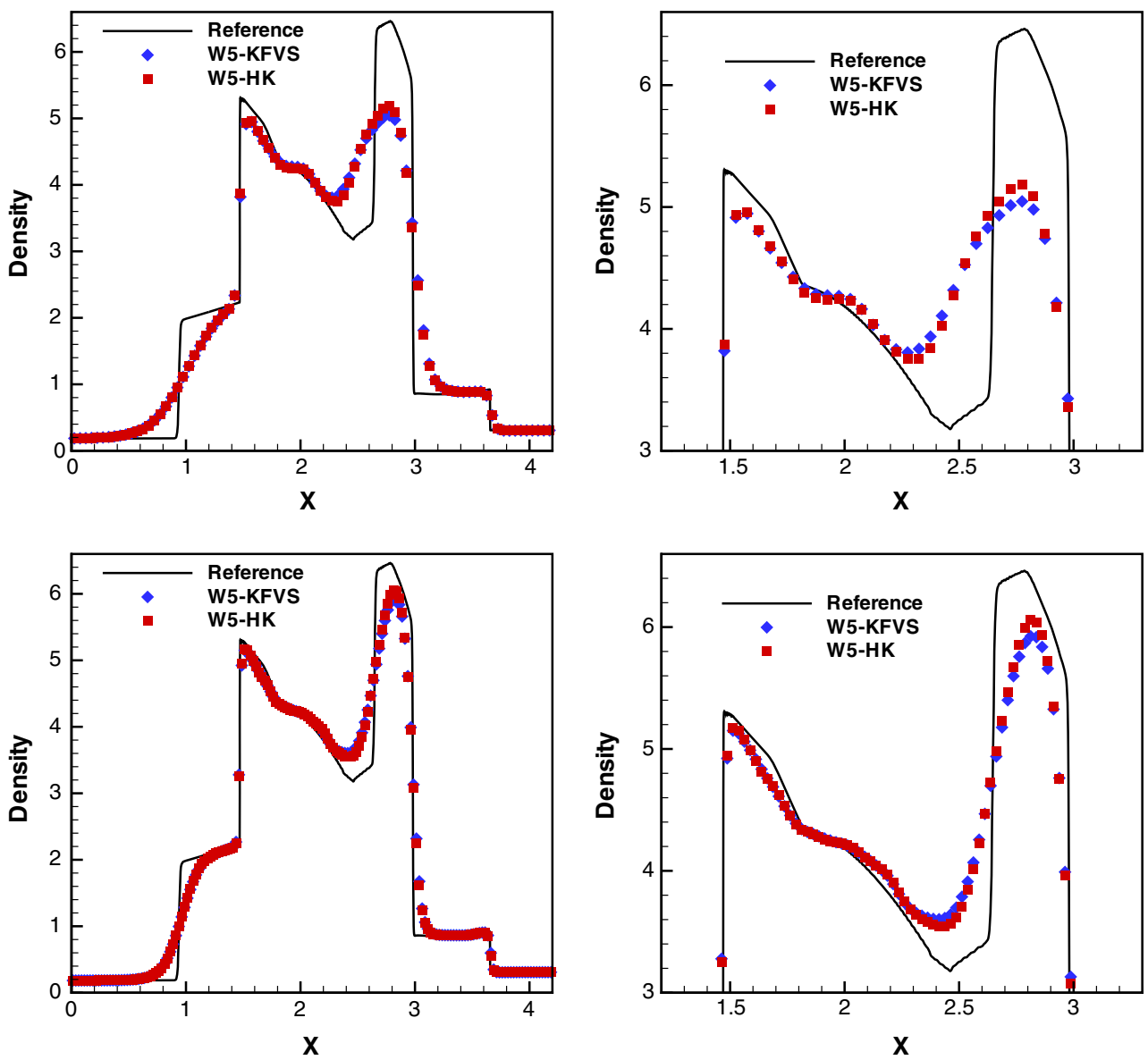

Figure 1. Blast wave problem, $N=200$ (top) and $N=400$ (bottom). The right figures are zoomed-in views of the left. The reference solution is obtained by the W5-HK scheme with 10000 cells.

Table II. Two-dimentional accuracy test.

\begin{tabular}{llcccccc}
\hline$N^{2}$ & Scheme & $L_{\text {error }}^{\infty}$ & Order & $L_{\text {error }}^{1}$ & Order & $L_{\text {error }}^{2}$ & Order \\
\hline $8^{2}$ & W5-KFVS & $3.70 \mathrm{E}-2$ & - & $2.25 \mathrm{E}-2$ & - & $2.50 \mathrm{E}-2$ & - \\
& W5-HK & $2.05 \mathrm{E}-2$ & - & $8.78 \mathrm{E}-3$ & - & $1.06 \mathrm{E}-2$ & - \\
$16^{2}$ & W5-KFVS & $2.66 \mathrm{E}-3$ & 3.80 & $1.49 \mathrm{E}-3$ & 3.92 & $1.72 \mathrm{E}-3$ & 3.86 \\
& W5-HK & $1.36 \mathrm{E}-3$ & 3.91 & $5.84 \mathrm{E}-4$ & 3.91 & $6.82 \mathrm{E}-4$ & 3.96 \\
$32^{2}$ & W5-KFVS & $1.52 \mathrm{E}-4$ & 4.13 & $6.28 \mathrm{E}-5$ & 4.57 & $7.41 \mathrm{E}-5$ & 4.54 \\
& W5-HK & $4.38 \mathrm{E}-5$ & 4.96 & $1.84 \mathrm{E}-5$ & 4.99 & $2.18 \mathrm{E}-5$ & 4.97 \\
$64^{2}$ & W5-KFVS & $6.96 \mathrm{E}-6$ & 4.45 & $2.11 \mathrm{E}-6$ & 4.90 & $2.69 \mathrm{E}-6$ & 4.78 \\
& W5-HK & $1.50 \mathrm{E}-6$ & 4.87 & $5.45 \mathrm{E}-7$ & 5.08 & $6.49 \mathrm{E}-7$ & 5.07 \\
$128^{2}$ & W5-KFVS & $2.41 \mathrm{E}-7$ & 4.85 & $6.60 \mathrm{E}-8$ & 5.00 & $8.58 \mathrm{E}-8$ & 4.97 \\
& W5-HK & $4.50 \mathrm{E}-8$ & 5.06 & $1.67 \mathrm{E}-8$ & 5.03 & $1.98 \mathrm{E}-8$ & 5.03 \\
$256^{2}$ & W5-KFVS & $6.89 \mathrm{E}-9$ & 5.13 & $2.00 \mathrm{E}-9$ & 5.04 & $2.56 \mathrm{E}-9$ & 5.07 \\
& W5-HK & $1.36 \mathrm{E}-9$ & 5.05 & $5.23 \mathrm{E}-10$ & 5.00 & $6.26 \mathrm{E}-10$ & 4.98 \\
$512^{2}$ & W5-KFVS & $1.76 \mathrm{E}-10$ & 5.29 & $5.70 \mathrm{E}-11$ & 5.13 & $7.12 \mathrm{E}-11$ & 5.17 \\
& W5-HK & $3.97 \mathrm{E}-11$ & 5.10 & $1.72 \mathrm{E}-11$ & 4.93 & $2.00 \mathrm{E}-11$ & 4.97 \\
\hline
\end{tabular}

We compute the solution up to $t=2$. The errors and convergence orders of density $\rho$ are shown in Table II, where it can be seen that the fifth-order convergence rate can be obtained by both schemes for 2D problems. Moreover, it is also observed that the W5-HK has smaller absolute errors than the W5-KFVS given the same cell size, which is consistent with the 1D accuracy test Example 4.1. 
In this paper, we mainly use the WENO reconstruction technique by Jiang and Shu [5], that is, Eqs. (3.3)-(3.5), for the proposed scheme. Other recently developed WENO reconstruction techniques, such as those in [17-19], can also work well for the proposed algorithm in our numerical experiments. As an illustration, we show the results obtained by using the WENO-Z reconstruction technique [18] for this 2D accuracy test problem in Table III, where we can see that it performs very well, and moreover the hybrid kinetic flux is more accurate than the KFVS flux.

Example 4.4 (Shock vortex interaction)

Next we solve the shock vortex interaction problem [5]. The computational domain is taken to be $[0,2] \times[0,1]$, a stationary Mach 1.1 shock is positioned at $x=0.5$. Its left upstream state is $\left(\rho, u_{1}, u_{2}, p\right)=(1,1.1 \sqrt{\gamma}, 0,1)$. A small vortex centered at $\left(x_{c}, y_{c}\right)=(0.25,0.5)$ is superimposed to the flow on the left of the shock. The vortex is a perturbation to the mean flow with the velocity $\left(u_{1}, u_{2}\right)$, temperature $T=p / \rho$ and entropy $S=\frac{p}{\rho^{\gamma}}$. The perturbation denoted by the tilde values is defined as

$$
\begin{aligned}
& \tilde{u}_{1}=\varepsilon \eta e^{\sigma\left(1-\eta^{2}\right)} \sin \theta, \quad \tilde{u}_{2}=-\varepsilon \eta e^{\sigma\left(1-\eta^{2}\right)} \cos \theta, \\
& \tilde{T}=-\frac{(\gamma-1) \varepsilon^{2} e^{2 \sigma\left(1-\eta^{2}\right)}}{4 \sigma \gamma}, \quad \tilde{S}=0,
\end{aligned}
$$

where $\eta=r / r_{c}$ with $r=\sqrt{\left(x-x_{c}\right)^{2}+\left(y-y_{c}\right)^{2}}, r_{c}$ is the critical radius for which the vortex has the maximum strength, $\varepsilon$ and $\sigma$ control the strength and decay rate of the vortex. In our computations, $\varepsilon=0.3, r_{c}=0.05$, and $\sigma=0.204$ are adopted.

The computational grid we use is $200 \times 100$. Figure 2 shows the pressure contours at $t=0.6$ by the W5-KFVS and W5-HK schemes. Figure 3 gives the pressure distributions along the horizontal line $y=0.5$ at $t=0.6$, where the reference solution is given by the W5-HK scheme with a refined mesh $2000 \times 1000$. From these figures, we can see that W5-HK can provide sharper shock transition than W5-KFVS for the same grid size.

In order to compare the efficiency for both W5-KFVS and W5-HK, we present the CPU time of simulations for two test cases in Table IV. As shown in the table, the CPU time for W5-HK is roughly $10-15 \%$ more than that for W5-KFVS in both one-dimensional and multi-dimensional simulations.

Table III. Two-dimentional accuracy test using the weighted essentially nonoscillatory-Z reconstruction [18].

\begin{tabular}{llllllll}
\hline$N^{2}$ & Scheme & $L_{\text {error }}^{\infty}$ & Order & $L_{\text {error }}^{1}$ & Order & $L_{\text {error }}^{2}$ & Order \\
\hline $8^{2}$ & Wz5-KFVS & $1.71 \mathrm{E}-2$ & - & $8.61 \mathrm{E}-3$ & - & $9.60 \mathrm{E}-3$ & - \\
& Wz5-HK & $5.97 \mathrm{E}-3$ & - & $2.77 \mathrm{E}-3$ & - & $3.50 \mathrm{E}-3$ & - \\
$16^{2}$ & Wz5-KFVS & $1.27 \mathrm{E}-3$ & 3.75 & $4.12 \mathrm{E}-4$ & 3.39 & $5.62 \mathrm{E}-4$ & 4.09 \\
& Wz5-HK & $2.39 \mathrm{E}-4$ & 4.64 & $1.26 \mathrm{E}-4$ & 4.46 & $1.43 \mathrm{E}-4$ & 4.61 \\
$32^{2}$ & Wz5-KFVS & $5.77 \mathrm{E}-5$ & 4.46 & $1.52 \mathrm{E}-5$ & 4.76 & $2.23 \mathrm{E}-5$ & 4.66 \\
& Wz5-HK & $7.47 \mathrm{E}-6$ & 5.00 & $4.11 \mathrm{E}-6$ & 4.94 & $4.61 \mathrm{E}-6$ & 4.96 \\
$64^{2}$ & Wz5-KFVS & $1.95 \mathrm{E}-6$ & 4.89 & $4.74 \mathrm{E}-7$ & 5.00 & $7.15 \mathrm{E}-7$ & 4.96 \\
& Wz5-HK & $2.35 \mathrm{E}-7$ & 4.99 & $1.30 \mathrm{E}-7$ & 4.98 & $1.45 \mathrm{E}-7$ & 4.99 \\
$128^{2}$ & Wz5-KFVS & $6.19 \mathrm{E}-8$ & 4.98 & $1.48 \mathrm{E}-8$ & 5.00 & $2.24 \mathrm{E}-8$ & 5.00 \\
& Wz5-HK & $7.30 \mathrm{E}-9$ & 5.01 & $4.08 \mathrm{E}-9$ & 4.99 & $4.57 \mathrm{E}-9$ & 4.99 \\
$256^{2}$ & Wz5-KFVS & $1.83 \mathrm{E}-9$ & 5.08 & $4.46 \mathrm{E}-10$ & 5.05 & $6.65 \mathrm{E}-10$ & 5.07 \\
& Wz5-HK & $2.30 \mathrm{E}-10$ & 4.99 & $1.30 \mathrm{E}-10$ & 4.97 & $1.45 \mathrm{E}-10$ & 4.98 \\
$5122^{2}$ & Wz5-KFVS & $6.18 \mathrm{E}-11$ & 4.89 & $1.49 \mathrm{E}-11$ & 4.90 & $2.22 \mathrm{E}-11$ & 4.90 \\
& Wz5-HK & $7.53 \mathrm{E}-12$ & 4.93 & $4.25 \mathrm{E}-12$ & 4.93 & $4.81 \mathrm{E}-12$ & 4.91 \\
\hline
\end{tabular}

(Wz5-KFVS, the 5th-order WENO-Z scheme [18] with the KFVS flux; Wz5-HK, the 5th-order WENO-Z scheme with the Hybrid Kinetic flux.) 

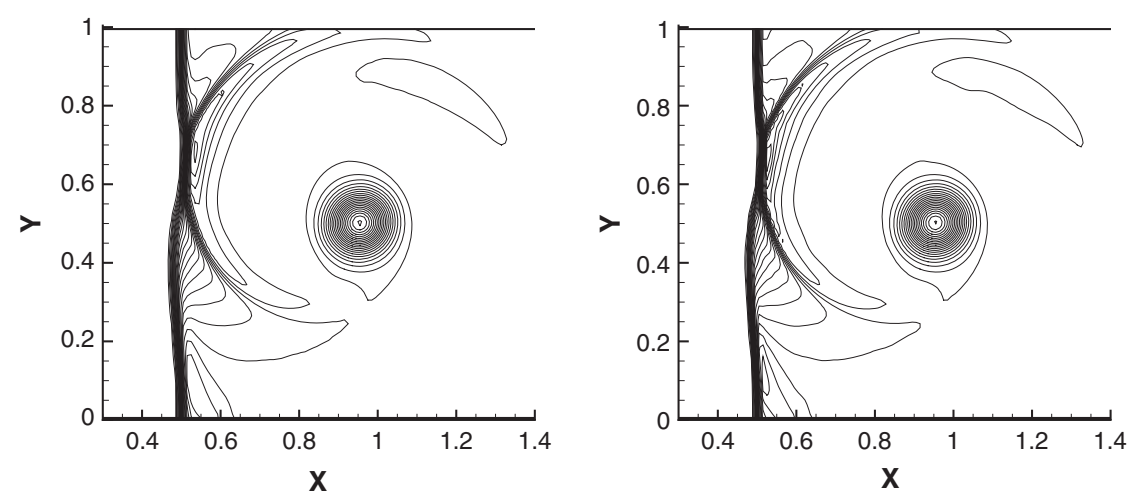

Figure 2. Shock vortex interaction problem, $\Delta x=\Delta y=\frac{1}{100}, t=0.6 .30$ equally spaced pressure contours from 1.02 to 1.32. Left: W5-KFVS; right: W5-HK.
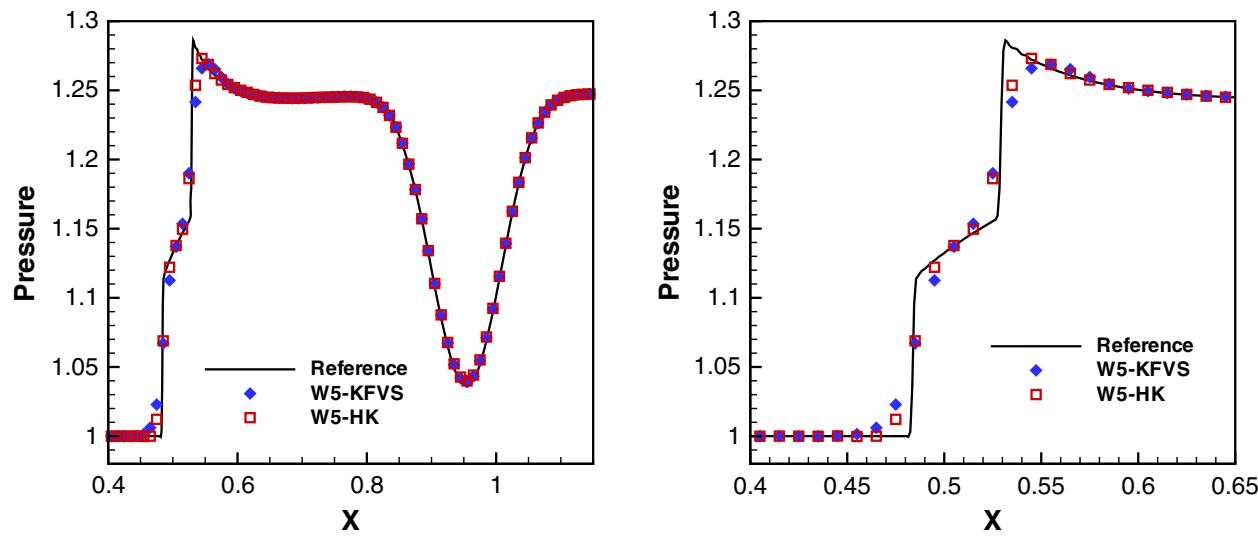

Figure 3. Shock vortex interaction problem, $\Delta x=\Delta y=\frac{1}{100}, t=0.6$. Pressure distributions along the central line $y=0.5$. The right figure is the zoomed-in view of the left. The reference solution is obtained by the W5-HK scheme with a refined mesh $2000 \times 1000$.

Table IV. The CPU time (in seconds) of simulations.

\begin{tabular}{lcc}
\hline Test case & W5-KFVS & W5-HK \\
\hline Blast wave problem (1D) & 1.90 & 2.18 \\
Shock vortex interaction (2D) & 9.59 & 10.70 \\
\hline
\end{tabular}

\subsection{Viscous flow problems}

Example 4.5 (Navier-Stokes shock structure)

In order to evaluate the performances of W5-KFVS and W5-HK in solving the Navier-Stokes equations, first, we consider the Navier-Stokes shock structure test [9]. The initial condition is set as a stationary shock with $M a=1.1, \rho=1, u=1$, and $\gamma=5 / 3$. The constant dynamic viscosity $\mu=2.5 \times 10^{-4}$ and the Prandtl number $P r=0.72$ are adopted. The analytic Navier-Stokes solution can be obtained by solving the ordinary differential equations [9].

Figure 4 shows the computed density distributions based on different mesh sizes. Good mesh convergence has been achieved for both W5-KFVS and W5-HK. Moreover, W5-HK performs overall better than W5-KFVS, which indicates that W5-HK is more accurate and less dissipative than W5-KFVS. 

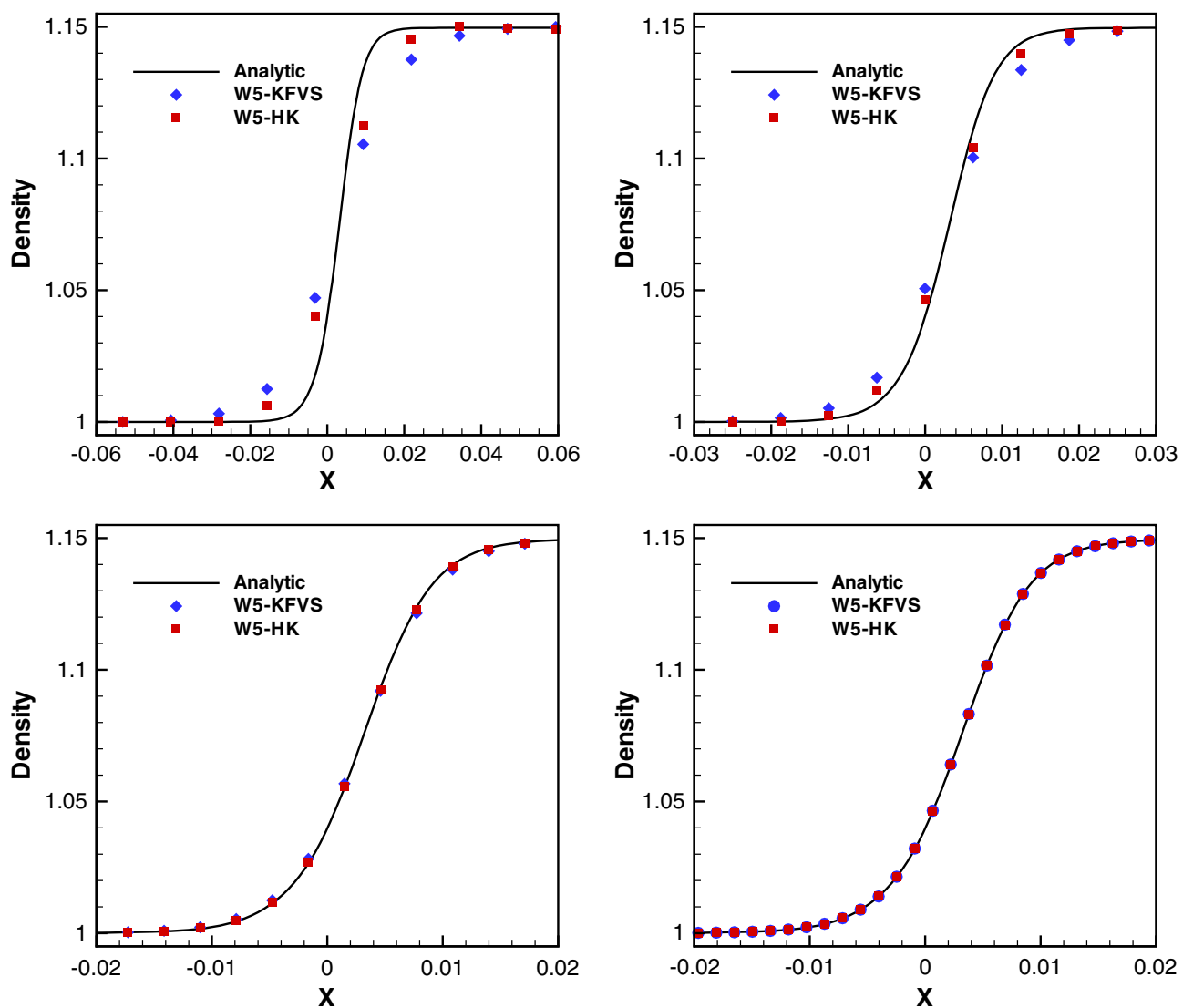

Figure 4. Navier-Stokes shock structure, $M a=1.1, \mu=0.00025$. Top to bottom and left to right: $\Delta x=$ $\frac{1}{80}, \frac{1}{160}, \frac{1}{320}, \frac{1}{640}$.

Example 4.6 (Couette flow)

The Couette flow is a typical test governed by the compressible Navier-Stokes equations; it is the laminar flow of a viscous fluid between two parallel plates, one of which is moving relatively to the other. The two-dimensional Navier-Stokes equations need to be solved although the physical flow is one-dimensional. In the following, we will consider two types of Couette flows.

Near incompressible Couette flow: With the bottom wall fixed, the top boundary is moving at a speed $U_{w}$ in the horizontal direction. The temperatures at the bottom and top are fixed with values $T_{0}$ and $T_{1}$, respectively. Under the assumption of constant viscosity and heat conduction coefficients and in the incompressible limit, a steady state analytic temperature distribution can be obtained

$$
\frac{T-T_{0}}{T_{1}-T_{0}}=\frac{y}{H}+\frac{\operatorname{Pr} U_{w}^{2}}{2 C_{p}\left(T_{1}-T_{0}\right)} \frac{y}{H}\left(1-\frac{y}{H}\right)
$$

where $H$ is the height of the channel, $\operatorname{Pr}$ is the Prandtl number, and $C_{p}$ is the specific heat ratio at constant pressure.

In the present study, we take $H=1, \rho=1, U_{w}=1, T_{0}=100 / 1.4, T_{1}=1.0002 T_{0}, \mu=$ $5 \times 10^{-3}, P r=0.72, \gamma=1.4$, and $C_{p}=3.5$. The Mach number corresponding to $U_{w}$ and $T_{0}$ is $M a=0.1$ in this test. Figure 5 shows the temperature distributions with $N=5,10$. The temperature profile is well captured by both W5-KFVS and W5-HK, which indicates that the heat flux can be accurately predicted. Furthermore, it can be clearly seen that W5-HK provides more accurate numerical solution than W5-KFVS with the same cell number $N=5$.

Compressible Couette flow: In the aformentioned near incompressible test, the velocity in the channel is almost linearly distributed. In the following, we are going to test W5-KFVS and W5-HK 

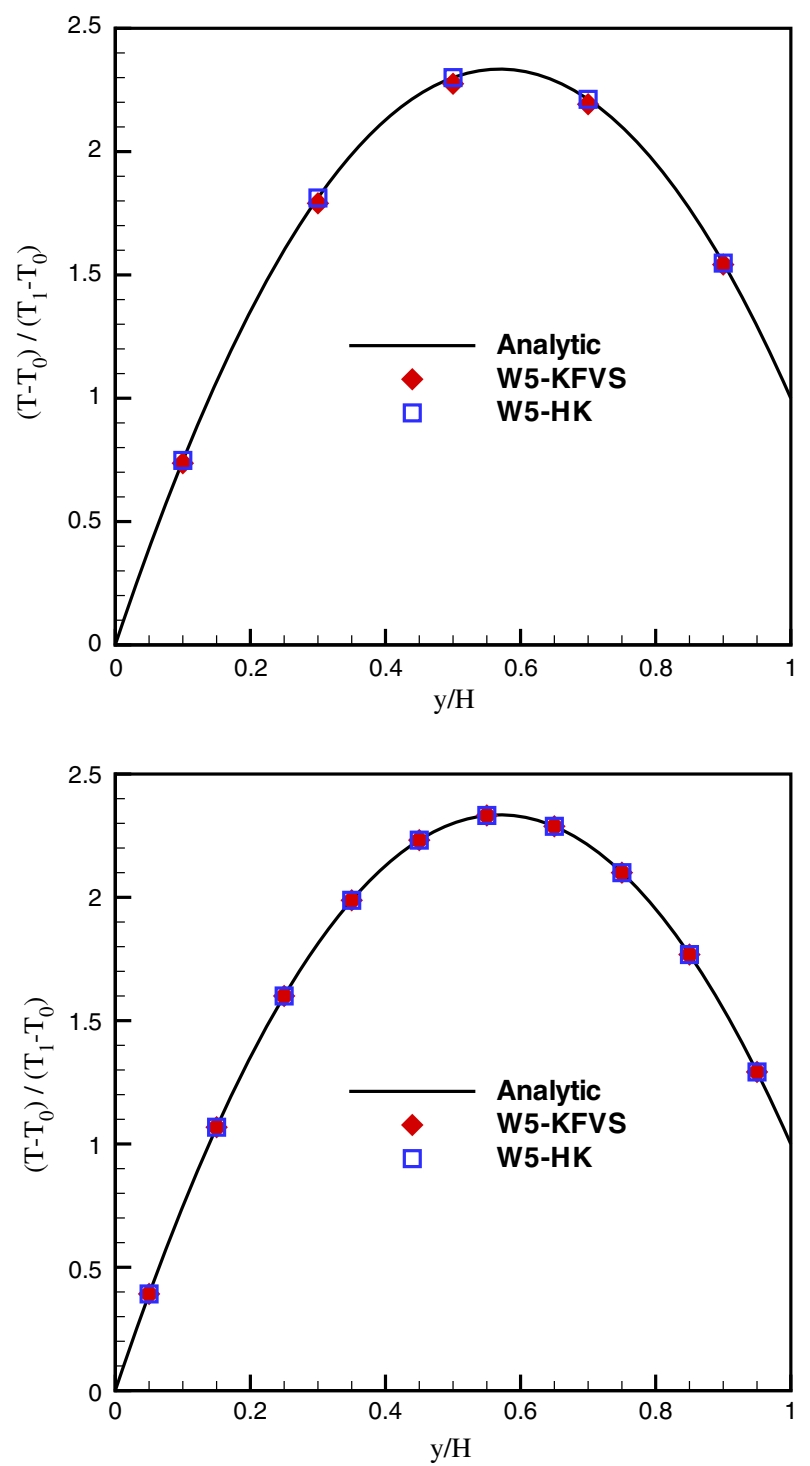

Figure 5. Temperature distributions for the near incompressible Couette flow, $N=5$ (top) and $N=10$ (bottom).

when the stress and heat flux are large. When the moving velocity of the upper wall $U_{w}$ is large enough, the compressibility of the fluid becomes appreciable. Under the conditions of $\mu \sim T$ and adiabatic lower wall boundary, there is an analytic solution in the compressible case [27]

$$
\frac{\tau_{w} y}{\mu_{\infty} U_{w}}=\frac{u}{U_{w}}+\operatorname{Pr} \frac{\gamma-1}{2} M a_{\infty}^{2}\left[\frac{u}{U_{w}}-\frac{1}{3}\left(\frac{u}{U_{w}}\right)^{3}\right]
$$

where $\tau_{w}$ is the shear stress at the top wall, $\mu_{\infty}$ is the dynamic viscosity coefficient corresponding to the top wall temperature $T_{1}$, and $M a_{\infty}$ is the Mach number associated with $U_{w}$ and $T_{1}$.

In our computations, we set $\gamma=1.4, P r=0.72$, and $\mu=5 \times 10^{-3} T / T_{1}$. The velocity distributions in the channel for $M a_{\infty}=1.5,3$ are shown in Figure 6, where we can see that the nonlinear velocity profiles are well predicted by both schemes with only five cells in the computational domain. Moreover, W5-HK does provide more accurate numerical solutions than W5-KFVS. 

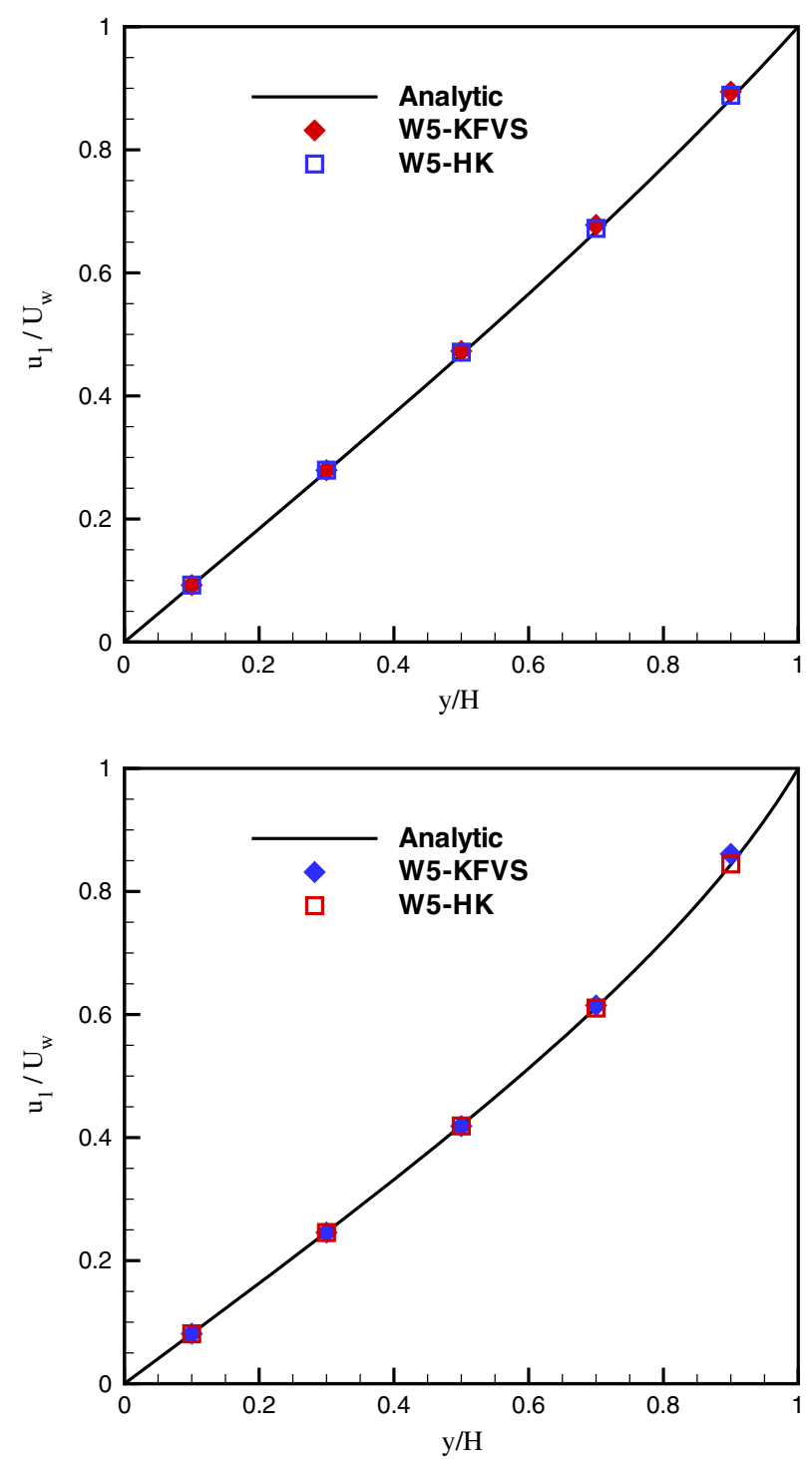

Figure 6. Velocity distributions for the compressible Couette flow, $M a=1.5$ (top) and $M a=3$ (bottom).

\section{CONCLUSIONS}

A hybrid kinetic WENO scheme is proposed for numerical simulations of inviscid and viscous flows, in which the effects of molecule collisions are included by constructing a hybrid kinetic numerical flux based on the WENO reconstruction technique.

Numerical experiments have demonstrated that the present method (W5-HK) is more accurate and less dissipative than the conventional scheme with the KFVS technique (W5-KFVS) for smooth flows; furthermore, the former can provide shaper discontinuity transition than the latter for flows with discontinuities (inviscid) or unresolved mesh conditions (viscous). In terms of the efficiency, the CPU time for W5-HK is roughly 10-15\% more than that for W5-KFVS in both one-dimensional and multi-dimensional simulations.

The present study indicates that the collisionless KFVS technique is intrinsically very dissipative, the cautious consideration of the influences of molecule collisions can really reduce the numerical dissipation of a high-order scheme. 


\section{ACKNOWLEDGEMENTS}

The author would like to thank Prof. Kun Xu for helpful discussions on the present work and the unknown referees for their constructive comments and suggestions, which greatly improve the manuscript.

\section{REFERENCES}

1. Harten A, Engquist B, Osher S, Chakravarthy S. Uniformly high order essentially non-oscillatory schemes III. Journal of Computational Physics 1987; 71:231-303.

2. Shu C-W, Osher S. Efficient implementation of essential non-oscillatory shock capturing schemes. Journal of Computational Physics 1988; 77:439-471.

3. Shu C-W, Osher S. Efficient implementation of essential non-oscillatory shock capturing schemes, II. Journal of Computational Physics 1989; 83:32-78.

4. Liu X, Osher S, Chan T. Weighted essentially non-oscillatory schemes. Journal of Computational Physics 1994; 115:200-212.

5. Jiang G, Shu C-W. Efficient implementation of weighted ENO schemes. Journal of Computational Physics 1996; 126:202-228.

6. Pullin DI. Direct simulation methods for compressible inviscid ideal gas flow. Journal of Computational Physics 1980; 34:231-244.

7. Mandal JC, Deshpande SM. Kinetic flux vector splitting for Euler equations. Computers \& Fluids 1994; 23:447-478.

8. Chou SY, Baganoff D. Kinetic flux-vector splitting for the Navier-Stokes equations. Journal of Computational Physics 1997; 130:217-230.

9. Xu K. A gas-kinetic BGK scheme for the Navier-Stokes equations and its connection with artificial dissipation and Godunov method. Journal of Computational Physics 2001; 171:289-335.

10. Xu K, Mao ML, Tang L. A multidimensional gas-kinetic BGK scheme for hypersonic viscous flow. Journal of Computational Physics 2005; 203:405-421.

11. Ohwada T, Kobayashi S. Management of the discontinuous reconstruction in kinetic schemes. Journal of Computational Physics 2004; 197:116-138.

12. Li QB, Xu K, Fu S. A high-order gas-kinetic Navier-Stokes solver. Journal of Computational Physics 2010; 229:6715-6731.

13. Liu N, Tang H. A high-order accurate gas-kinetic scheme for one- and two-dimensional flow simulation. Communications in Computational Physics 2014; 15:911-943.

14. Kumar G, Girimaji SS, Kerimo J. WENO-enhanced gas-kinetic scheme for direct simulations of compressible transition and turbulence. Journal of Computational Physics 2013; 234:499-523.

15. Xuan LJ, Xu K. A new gas-kinetic scheme based on analytical solutions of the BGK equation. Journal of Computational Physics 2013; 234:524-539.

16. Luo J, Xu K. A high-order multidimensional gas-kinetic scheme for hydrodynamic equations. Science China Technological Sciences 2013; 56:2370-2384.

17. Henrick AK, Aslam TD, Powers JM. Mapped weighted essentially non-oscillatory schemes: achieving optimal order near critical points. Journal of Computational Physics 2005; 207:542-567.

18. Borges R, Carmona M, Costa B, Don WS. An improved weighted essentially non-oscillatory scheme for hyperbolic conservation laws. Journal of Computational Physics 2008; 227:3191-3211.

19. Fan P, Shen YQ, Tian BL, Yang C. A new smoothness indicator for improving the weighted essentially nonoscillatory scheme. Journal of Computational Physics 2014; 269:329-354.

20. Steger JL, Warming R. Flux vector splitting of the inviscid gas dynamic equation with application to finite difference methods. Journal of Computational Physics 1981; 40:263-293.

21. Xu K, Jameson A. Gas-kinetic relaxation (BGK-type) schemes for the compressible Euler equations. 12th AIAA Computational Fluid Dynamics Conference AIAA-95-1736, San Diego, California, 19-22 June 1995.

22. Ohwada T, Fukata S. Simple derivation of high-resolution schemes for compressible flows by kinetic approach. Journal of Computational Physics 2006; 211:424-447.

23. Liu H, Xu K. A Runge-Kutta discontinuous Galerkin method for viscous flow equations. Journal of Computational Physics 2007; 224:1223-1242.

24. Roe PL. Approximate Riemann solvers, parameter vectors, and difference schemes. Journal of Computational Physics 1981; 43:357-372.

25. Guo Z, Liu H, Luo LS, Xu K. A comparative study of the LBE and GKS methods for 2D near incompressible laminar flows. Journal of Computational Physics 2008; 227:4955-4976.

26. Woodward P, Colella P. The numerical simulation of two-dimensional fluid flow with strong shocks. Journal of Computational Physics 1984; 54:115-173.

27. Liepmann HW, Roshko A. Elements of Gasdynamics. Wiley: New York, 1957. 